\title{
Fixed Windows in Fractional Fourier Domain
}

\author{
Rahul Pachauri, Rajiv Saxena \\ Jaypee University of Engineering \& Technology, Guna (M.P.), India \\ pachauri.123@gmail.com, rajiv.saxena@juet.ac.in \\ Sanjeev N. Sharma \\ Samrat Ashok Technological Institute, Vidisha (M.P.), India \\ sanjeev_n_sharma@rediffmail.com
}

\begin{abstract}
In this study, some mathematical relations have been derived for the useful parameters of fixed window functions in fractional Fourier transform (FRFT) domain. These reported expressions are also verified with the simulation studies. The FRFT provides an important extension to conventional Fourier transform with an additional degree of freedom by which these parameters of window functions can be controlled while inherent time domain behavior of the windows remains intact. The behavior of fixed windows on timefrequency plane has been varied by varying the FRFT order. The obtained variability in the window functions has been applied in the designing of FIR filters.
\end{abstract}

Index Terms - Fractional Fourier Transform, Null Bandwidth, Half Main Lobe Width, Maximum Side Lobe Level.

\section{INTRODUCTION}

Several applications of window functions have been reported in the fields of signal processing and communications, such as digital filter design, spectrum estimation, beam-forming, and speech processing [1]. The useful parameters of window functions for these applications include Half Main Lobe Width (HMLW), Normalized Band Width (NBW), Maximum Side Lobe Level (MSLL), and Side Lobe Fall of Rate (SLFOR). A concise analysis of these parameters and their properties has been presented by Harris [2]. In [2], Harris has described time domain and frequency domain parameters of window functions. Frequency domain behavior of window functions has been studied by mapping the time domain window function in frequency domain using Fourier Transform (FT).

In this study, characterization of window function parameter has been carried out in fractional Fourier domain which is a generalization of the FT. The Fractional Fourier Transform (FRFT) with an additional adjustable parameter, FRFT order $(a)$, makes it more flexible and is having numerous applications in many areas [3-5], where as its implementation cost is at par with FT. [5]-

The continuous-time FRFT of a signal $x(t)$ is given as

$$
X_{\alpha}(u)=\int_{-\infty}^{\infty} x(t) K_{\alpha}(t, u) d t
$$

where, the transformation kernel $K_{\alpha}(t, u)$ of the FRFT is defined as-

$$
K_{\alpha}(t, u)=\left\{\begin{array}{lr}
\sqrt{\frac{1-i \cot (\alpha)}{2 \pi} e^{i}} e^{\left(\frac{u^{2}+t^{2}}{2}\right) \cot (\alpha)-\text {-iutcosec }(\alpha)} & \text { if } \alpha \neq n \pi \\
\delta(t-u) & \text { if } \alpha=2 n \pi \\
\delta(t+u) & \text { if }(\alpha+\pi)=2 n \pi
\end{array}\right.
$$

where, $\alpha=a \pi / 2$ is the rotation angle of the transformed signal of the FRFT.

Prior to this work, analysis of some window functions in FRFT domain has been carried out by Kumar et al. [6], and it has been observed that the window parameters can be varied by changing the FRFT order. However, no mathematical relationships have been established between window parameters and FRFT order in [6]. It has been shown in [6] that when FRFT order is decreased from 1 to 0, the MSLL and SLFOR increase while HMLW decreases. Same methodology has been used earlier in [7] to tune the Transition Bandwidth (TBW) of Kaiser Window [2] and PC6 window [8] based FIR filters using FRFT. It has been shown in [7] that when the FRFT parameter is decreased the Transition Bandwidth (TBW) of the window based FIR filter decreases. Variations in the better TBW have been obtained without re-computation of filter impulse response coefficients. However, the applicability of this phenomenon has been demonstrated in [7] only for lower order FIR filters.

In this paper, analysis of rectangular (Dirichlet), generalized 2 -term cosine (Hanning / raised cosine / Hamming), Blackman and triangular window functions in FRFT domain has been reported. These windows fall in the fixed windows category. Relationships between window function parameters and FRFT order have been established. These relationships have been utilized in the design of fixed window based FIR filters.

The rest of paper is organized as follows: In Section-2 characterization of windows in FRFT domain has been carried out. Limiting values for the length of window 
functions, about which their NBW shrinks and expands, have been determined in Section-3. This analysis is based on the principle of uncertainty product for the window function [9]. The relationships established in previous sections have been used in Section-4 for the designing of FIR filters. Finally, the paper is concluded in Section-5.

\section{CHARACTERIZATION OF WINDOW FUNCTIONS IN FRACTIONAL DOMAIN}

The mathematical analysis of fixed windows in the FRFT domain has been carried out in this section to establish the relationships between window parameters and FRFT order. Plot shown in Figure 1 illustrate the four frequency domain parameters for a window function, viz. MSLL, SLFOR, HMLW, and NBW, for which the relationships in fractional Fourier domain have been established. Characterization has been carried out for four fixed windows- (a) Rectangular (b) Generalized Hamming (c) Blackman (d) Triangular window, as given in following section-

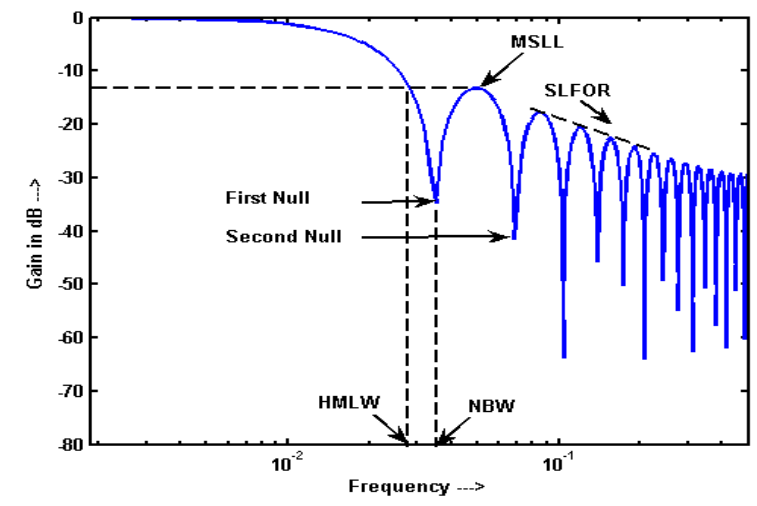

Figure 1. Plot for illustrating the definition of the parameters of window function

\section{A. Rectangular Window $(R W)$}

The time domain expression for a RW is given as-

$$
w_{R}(t)=\left\{\begin{array}{lr}
1, & t \leq \frac{\tau}{2} \\
0, & \text { elsewhere }
\end{array}\right.
$$

The FRFT domain expression $W_{R}(u)$ of RW (A1.4) has been included in Appendix-1.

The magnitude of $W_{R}(u)$ for this window is given by-

$$
\begin{aligned}
& \left|W_{R}(u)\right|_{R}=\left|\sqrt{\frac{1-i \cot (\alpha)}{8}}\right| \times \\
& \mid \operatorname{erfi}\left[\frac{1+i}{2} \sqrt{\cot (\alpha)}\left\{\frac{\tau}{2}-u \sec (\alpha)\right\}\right] \\
& -\operatorname{erfi}\left[\frac{1+i}{2} \sqrt{\cot (\alpha)}\left\{-\frac{\tau}{2}-u \sec (\alpha)\right\} \mid\right.
\end{aligned}
$$

The mathematical relationships for NBW, MSLL, and HMLW in fractional domain have been obtained by differentiating the fractional domain response of the window functions. This follows from the mathematical results that the position of maxima and minima of any function can be obtained by the differentiation of that function.

The plots for the magnitude of $W_{R}(u)$ and its differentiation with respect to $u$ for $\alpha=0.4$ are shown in Figure 2 and 3 respectively. From these figures it follows that the zeros of $\frac{d}{d u}\left|W_{R}(u)\right|$ occur at the positions similar to the null positions of $\left|W_{R}(u)\right|$. Thus, the position of the window's FRFT nulls can be obtained by differentiating (4) with respect to $u$ and equating it to zero as given in Appendix-2

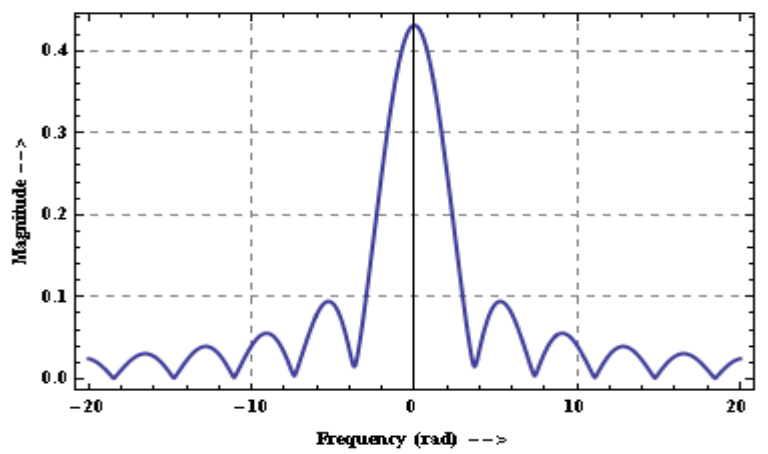

Figure 2. FRFT of rectangular window for $\tau=1$ and $a=0.4$

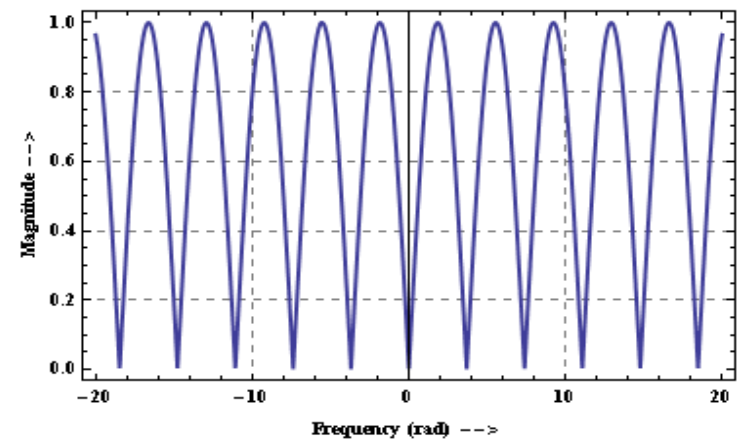

Figure 3. Plot for the differentiation of $\left|W_{\alpha}(u)\right|$ with respect to $u$

The position of nulls of $\left|W_{R}(u)\right|$ for various values of $n$ are given by (A2.6) and for $n=1$ it determines the 
NBW of the RW. Thus, the NBW in FRFT domain for $\mathrm{RW}\left(u_{N R}\right)$ is -

$$
u_{N R}=\left|\frac{2 \pi \sin (\alpha)}{\tau}\right|
$$

The coefficient of $\sin (\alpha)$ in the above expression defines the NBW of continuous time RW in Fourier domain. On the other hand, for $N$-point discrete time RW the transition width of mainlobe in FT domain is given by $4 \pi / N$ [10] which can be normalized to define NBW as $1 / N$.

TABLE I. DISCRETE RW PARAMETERS FOR $N=25$ AND $M=2048$

\begin{tabular}{|c|c|c|c|c|c|c|}
\hline \multirow{2}{*}{$\begin{array}{r}\text { FRFT } \\
\text { Order, } a\end{array}$} & \multicolumn{2}{|l|}{ NBW } & \multicolumn{2}{|l|}{ HMLW } & \multicolumn{2}{|c|}{ MSLL (dB) } \\
\hline & SR & AR & SR & AR & SR & AR \\
\hline 1.0 & 0.04 & 0.04 & 0.0323 & 0.0324 & -13.23 & -13.2627 \\
\hline 0.9 & 0.0396 & 0.0395 & 0.0320 & 0.0320 & -13.21 & -13.2618 \\
\hline 0.8 & 0.0381 & 0.038 & 0.0310 & 0.0300 & -13.20 & -13.2591 \\
\hline 0.7 & 0.0357 & 0.0356 & 0.0290 & 0.0289 & -13.19 & -13.2540 \\
\hline 0.6 & 0.0323 & 0.0324 & 0.0263 & 0.0262 & -13.15 & -13.2450 \\
\hline 0.5 & 0.0284 & 0.0283 & 0.0230 & 0.0229 & -13.10 & -13.2293 \\
\hline 0.4 & 0.0236 & 0.0235 & 0.0200 & 0.0190 & -13.01 & -13.1997 \\
\hline 0.3 & 0.0183 & 0.0182 & 0.0150 & 0.0147 & -12.79 & -13.1355 \\
\hline 0.2 & 0.0120 & 0.0124 & 0.0110 & 0.0100 & -12.20 & -12.955 \\
\hline 0.1 & 0.0060 & 0.0063 & 0.0052 & 0.0051 & -9.380 & -12.070 \\
\hline
\end{tabular}

Thus, the above relation expresses $u_{N R}$ in terms of NBW in Fourier domain, i.e., $u_{F T}$ as-

$$
u_{N R}=\left|u_{F T} \sin (\alpha)\right|
$$

where,

$$
u_{F T}=\left\{\begin{array}{lr}
\frac{2 \pi}{\tau}, & \text { for continuous }- \text { time } R W \\
\frac{1}{N}, & \text { for discrete }- \text { time } R W \text { of } N \text { samples }
\end{array}\right.
$$

It has been observed from the simulation studies that the MSLL occurs at 1.425 times of NBW as shown in Figure 4 (shown from 0 to 0.2 in place of actual range 0 to 0.5 for the sake of clarity). Therefore, the expression for the position of MSLL of RW can be written as-

$$
u_{M R}=\left|1.425 u_{F T} \sin (\alpha)\right|
$$

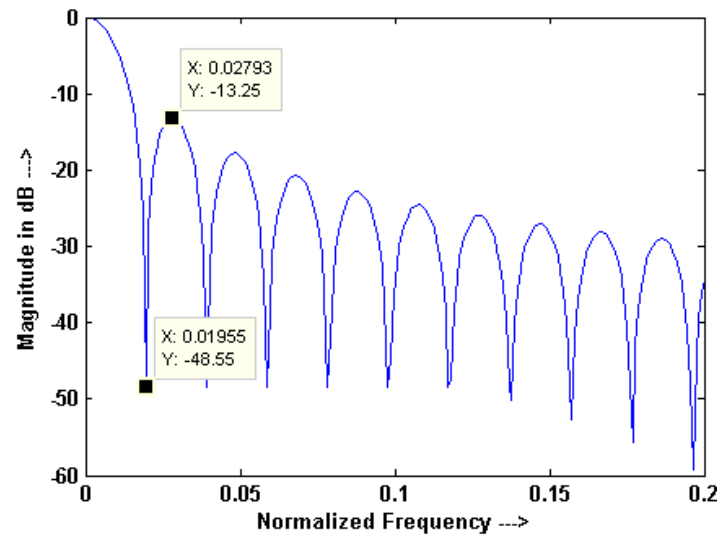

Figure 4. Plot for the position of NBW and MSLL of RW for window length $N=51$
The magnitude of MSLL has been obtained by substituting (7) in (4) as given by (8)-

$|M S L L|_{R}=\left|\sqrt{\frac{1-i \cot (\alpha)}{8}}\right| \times$

$\left|\begin{array}{l}\operatorname{erfi}\left[\frac{1+i}{2} \sqrt{\cot (\alpha)}\left\{0.5 \tau-1.425 u_{F T} \tan (\alpha)\right\}\right] \\ -\operatorname{erfi}\left[\frac{1+i}{2} \sqrt{\cot (\alpha)}\left\{-0.5 \tau-1.425 u_{F T} \tan (\alpha)\right\}\right]\end{array}\right|$

It has been shown in $[11,12]$ that HMLW of RW is 0.81 times of NBW. Therefore, the HMLW in FRFT domain, $u_{H R}$, can be expressed as-

$$
u_{H R}=\left|0.81 u_{F T} \sin (\alpha)\right|
$$

The obtained Analytical Results (AR) for NBW, MSLL and HMLW has been verified using Simulation Results (SR). The recorded values for these parameters are shown in Tables I.

The expressions for other fixed windows have been also obtained in FRFT domain by following the same methodology adopted in RW.

B. Generalized Hamming Window (GHW)

The GHW is defined in time domain as-

$$
w_{H}(t)=\left\{\begin{array}{lr}
b+(1-b) \cos (2 \pi t), & |t| \leq 0.5 \\
0, & \text { elsewhere }
\end{array}\right.
$$

For $b=0.5$, Hanning window results while for $b=$ 0.54 , Hamming window results. 
TABLE II. DISCRETE HW PARAMETERS FOR N = 35 AND M = 2048

\begin{tabular}{|c|l|l|l|l|l|l|}
\hline \multirow{2}{*}{$\begin{array}{c}\text { FRFT } \\
\text { Order, } a\end{array}$} & \multicolumn{2}{|l|}{ NBW } & \multicolumn{2}{l|}{ HMLW } & \multicolumn{2}{l|}{ MSLL $(\mathrm{dB})$} \\
\cline { 2 - 8 } & SR & AR & SR & AR & AR \\
\hline 1.0 & 0.0590 & 0.0571 & 0.055 & 0.0534 & -31.47 & -31.4743 \\
0.9 & 0.0581 & 0.0564 & 0.054 & 0.0528 & -31.44 & -31.4717 \\
0.8 & 0.0562 & 0.0543 & 0.052 & 0.0500 & -31.35 & -31.4635 \\
0.7 & 0.0528 & 0.0500 & 0.048 & 0.0476 & -31.16 & -31.4478 \\
0.6 & 0.0480 & 0.0462 & 0.045 & 0.0432 & -30.85 & -31.4206 \\
0.5 & 0.0422 & 0.0400 & 0.039 & 0.0378 & -30.29 & -31.3731 \\
0.4 & 0.0350 & 0.0336 & 0.029 & 0.0310 & -29.18 & -31.2843 \\
$*$ & & & & & & \\
\hline
\end{tabular}

The fractional domain expression of the GHW (A3.1) has been obtained by following the similar steps of RW and given in Appendix-3.

Since the NBW of GHW $\left(u_{N H}\right)$ is twice that of NBW of RW $\left(u_{R H}\right)[10,13]$. Therefore, using (6) $u_{N H}$ can be expressed as-

$$
u_{N H}=\left|u_{F T} \sin (\alpha)\right|
$$

where,

$u_{F T}=\left\{\begin{array}{lr}\frac{4 \pi}{\tau}, & \text { for continuous -time } G H W \\ \frac{2}{N}, & \text { for discrete -time GHW of } N \text { samples }\end{array}\right.$

It has been observed that the MSLL of GHW occurs at the 1.175 and 2.135 times of NBW for $b=0.5$ and 0.54 respectively as shown in Figure 5 . Thus, it can be written as-

$$
u_{M H}=\left\{\begin{array}{l}
\left|1.175 u_{F T} \sin (\alpha)\right|, \quad \text { when } b=0.5 \\
|| 2.135 u_{F T} \sin (\alpha) \mid, \quad \text { when } b=0.54
\end{array}\right.
$$

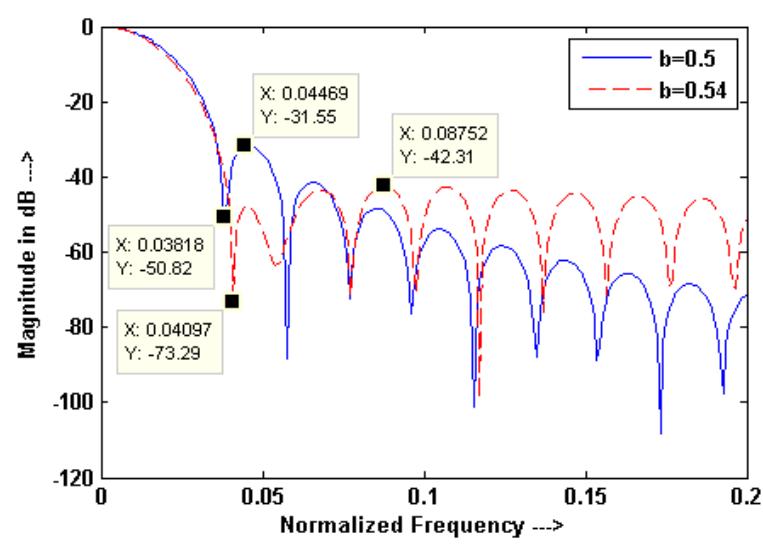

Figure 5. Plot for the position of NBW and MSLL of GHW for window length $N=51$
The expression for the MSLL in FRFT domain of GHW with $b=0.5$ has been obtained by substituting (12) in (A3.1) and given by (A3.2).

It has been shown in $[11,12]$ that the HMLW is at the 0.935 and 0.955 times of NBW for $b=0.5$ and 0.54 respectively. Therefore, it can be expressed in FRFT domain as-

$u_{H H}=\left\{\begin{array}{l}\left|0.935 u_{F T} \sin (\alpha)\right|, \quad \text { when } b=0.5 \\ \left|0.955 u_{F T} \sin (\alpha)\right|, \quad \text { when } b=0.54\end{array}\right.$

The obtained expressions for NBW, MSLL and HMLW of GHW have been verified with simulation studies. The recorded values for Hanning Window (HW) are shown in Tables II.

\section{Blackman Window $(B W)$}

The expression for the $\mathrm{BW}$ in time domain is given as-

$w_{B}(t)=\left\{\begin{array}{lr}0.42+0.5 \cos (2 \pi t)+0.08 \cos (4 \pi t),|t| \leq 0.5 \\ 0, \quad \text { elsewhere }\end{array}\right.$

The expression for the BW (A4.1) in FRFT domain is given in Appendix-4.

Since the NBW of BW $\left(u_{N B}\right)$ is thrice that of NBW of RW $\left(u_{R H}\right)[10,13]$. Therefore, using (6) $u_{N B}$ can be expressed as-

$u_{N B}=\left|u_{F T} \sin (\alpha)\right|$

where,

$u_{F T}=\left\{\begin{array}{lr}\frac{6 \pi}{\tau}, & \text { for continuous }- \text { time } B W \\ \frac{3}{N}, & \text { for discrete }- \text { time } B W \text { of } N \text { samples }\end{array}\right.$

It has been observed that the position of MSLL of BW is 1.18 times of NBW as shown in Figure 6. Thus, it can be defined in FRFT domain as- 


$$
u_{M B}=\left|1.18 u_{F T} \sin (\alpha)\right|
$$

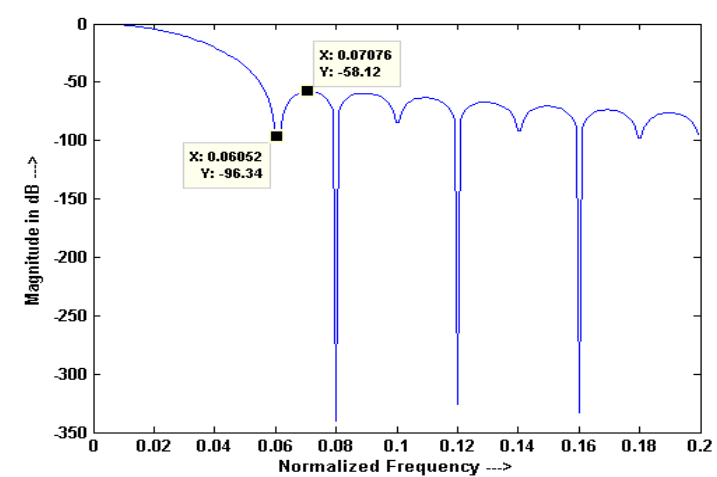

Figure 6. Plot for the position of NBW and MSLL of BW for window length $\mathrm{N}=51$
The FRFT domain relationship for the MSLL of BW (A4.2) has been obtained by substituting (16) in (A4.1) and given in Appendix-4.

The position of HMLW for BW [11, 12] in FRFT domain can be written as-

$$
u_{H B}=\left|0.94 u_{F T} \sin (\alpha)\right|
$$

The obtained expressions for NBW, MSLL and HMLW of BW have been verified with simulation results and recorded values are included in Tables III.

\begin{tabular}{|c|c|c|c|c|c|c|}
\hline \multirow{2}{*}{$\begin{array}{l}\text { FRFT } \\
\text { Order, } a\end{array}$} & \multicolumn{2}{|l|}{ NBW } & \multicolumn{2}{|l|}{ HMLW } & \multicolumn{2}{|l|}{ MSLL (dB) } \\
\hline & SR & $\mathrm{AR}$ & SR & $\mathrm{AR}$ & SR & $\mathrm{AR}$ \\
\hline 1.0 & 0.0590 & 0.0571 & 0.0480 & 0.0465 & -26.32 & -26.5325 \\
\hline 0.9 & 0.0576 & 0.0564 & 0.0473 & 0.0459 & -26.32 & -26.5323 \\
\hline 0.8 & 0.0552 & 0.0543 & 0.0453 & 0.0442 & -26.33 & -26.5353 \\
\hline 0.7 & 0.0514 & 0.0509 & 0.0431 & 0.0414 & -26.36 & -26.5351 \\
\hline 0.6 & 0.0473 & 0.0462 & 0.0396 & 0.0376 & -26.46 & -26.5383 \\
\hline 0.5 & 0.0421 & 0.0404 & 0.0358 & 0.0329 & -26.45 & -26.5439 \\
\hline 0.4 & 0.0359 & 0.0336 & 0.0315 & 0.0273 & -26.56 & -26.5545 \\
\hline 0.3 & 0.0307 & 0.0259 & 0.0261 & 0.0211 & -26.80 & -26.5777 \\
\hline 0.2 & 0.0229 & 0.0177 & 0.0198 & 0.0144 & -27.38 & -26.6443 \\
\hline
\end{tabular}

TABLE III. DISCRETE TW PARAMETERS FOR N = 35, M = 1024

TABLE IV. DISCRETE BW PARAMETERS FOR N = 45 AND M = 1024

\begin{tabular}{|c|c|c|c|c|c|c|}
\hline \multirow{2}{*}{$\begin{array}{c}\text { FRFT } \\
\text { Order, } a\end{array}$} & \multicolumn{2}{|c|}{ NBW } & \multicolumn{2}{|c|}{ HMLW } & \multicolumn{2}{c|}{ MSLL (dB) } \\
\cline { 2 - 7 } & SR & AR & SR & AR & SR & AR \\
0.9 & 0.0683 & 0.0667 & 0.0641 & 0.0627 & -58.12 & -58.1115 \\
0.8 & 0.0702 & 0.0658 & 0.0642 & 0.0619 & -58.14 & -58.1124 \\
0.7 & 0.0688 & 0.0634 & 0.0644 & 0.0596 & -58.21 & -58.1155 \\
0.6 & 0.0655 & 0.0594 & 0.0624 & 0.0558 & -58.27 & -58.1212 \\
& 0.0602 & 0.0539 & 0.0575 & 0.0507 & -58.19 & -58.1311 \\
$* .5$ & 0.0471 & 0.0530 & 0.0443 & 0.0514 & -57.45 & -58.1480 \\
* & & & & & \\
\hline
\end{tabular}

\section{Triangular Window (TW)}

The expression for the TW in time domain is given as-

$$
w_{T}(t)=\left\{\begin{array}{lr}
1-\frac{2|t|}{\tau}, & |t| \leq \frac{\tau}{2} \\
0, & \text { elsewhere }
\end{array}\right.
$$

The FRFT domain expression of TW (A5.1) is given in Appendix-5.
Since the NBW of TW $\left(u_{N T}\right)$ is twice that of NBW of RW $\left(u_{R H}\right)[10,13]$. Therefore, using (6) $u_{N T}$ can be expressed as-

$$
u_{N T}=\left|u_{F T} \sin (\alpha)\right|
$$

where,

$u_{F T}=\left\{\begin{array}{lr}\frac{4 \pi}{\tau}, & \text { for continuous - time window } \\ \frac{2}{N}, & \text { for discrete - time } T W \text { of } N \text { samples }\end{array}\right.$ 
Again, it has been observed by the simulation studies that the position of MSLL of this window is 1.45 times of NBW as shown in Figure 7. Therefore, it can be defined in FRFT domain as-

$$
u_{M T}=\left|1.45 u_{F T} \sin (\alpha)\right|
$$

The fractional domain expression for the MSLL of TW has been obtained by substituting (20) in (A5.1) and is given by (A5.2) as shown in Appendix-5.

The position of HMLW of TW $[11,12]$ can be written as-

$$
u_{H T}=\left|0.815 u_{F T} \sin (\alpha)\right|
$$

The derived expressions for NBW, MSLL and HMLW of have been verified with simulation results. The recorded values are included in Tables IV.

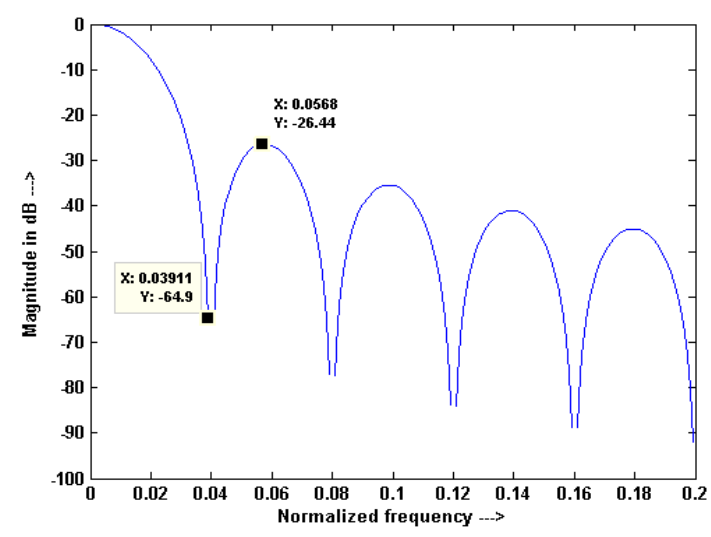

Figure 7. Plot for the position of NBW and MSLL of TW for window length $N=51$

\section{UNCERTAINTY PRODUCT IN FRACTIONAL DOMAIN}

It is easy to verify from that the FRFT holds an identity operator i.e. signal attains fully time domain behavior for $a=0$ while it is conventional FT i.e. signal jumps into frequency domain completely for $a=1$. If the FRFT order $a$ lies between these two ranges signal will be composed of frequency and time components both. In FT domain, the NBW is having inverse relationship to the window length and the product of these two parameters remains constant for every window function. This property of window functions can be thought of as the uncertainty principle [9] according to which a function in time domain and its FT cannot both be highly concentrated. If the window length is small the NBW is large and at particular window length these two parameters become equal and further increment from this window length NBW becomes smaller than the window length. This property of window functions has been exploited in this work to analyze the behavior of windows for below and above of this particular window length in FRFT domain. It has been observed in simulation studies that the NBW shrinks for small window length and expands for large window length when the FRFT order is varied from 1 to zero because frequency plane moves toward time plane i.e. window length. Thus, the FRFT provides another parameter to vary the NBW without varying the window length.

It has been observed by simulation studies that the time spread of $M$ zero padded discrete windows of length $N$ equals to the main lobe width of window function at particular window length (limiting length, $N$ ) for $a=1$. To find this limiting length $N$, time domain and frequency domain spread of RW has been shown in Figure 8 .
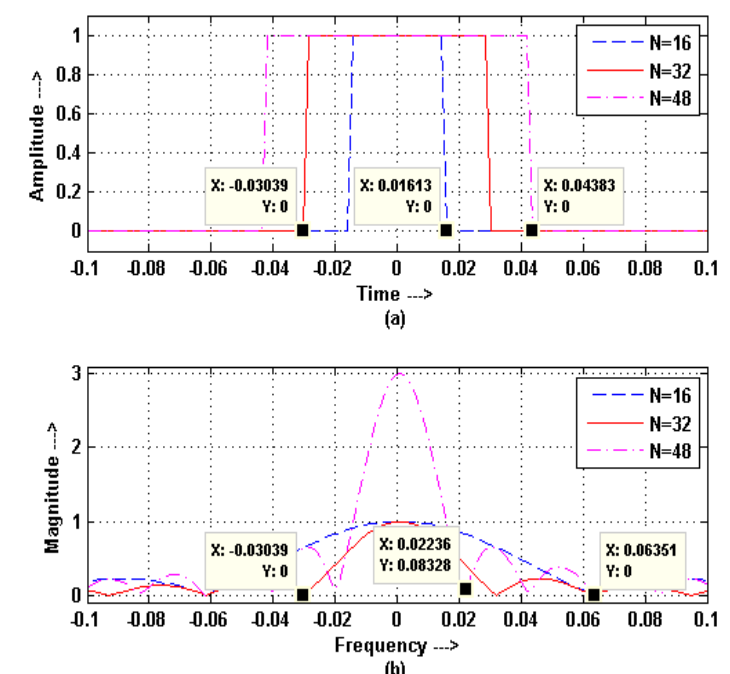

Figure 8. Expanded view of RW (a) time domain (b) frequency domain for different length $\mathrm{N}$ and $\mathrm{M}=512$ with $\mathrm{a}=$ 1

The X-axis of both the domains of $N$ sampled and $M$ zero padded RW has been normalized in the same range i.e. -0.5 to 0.5 however, it has been shown only in the range of -0.1 to 0.1 for the sake of clarity. The non-zero time spread of the normalized window is given by $(N+1) /(M+N)$ and $N /(M+N)$ for $N$ is odd and even respectively. The range of main lobe width of RW in the normalized frequency domain spread is given by $-1 / N$ to $1 / N$. Thus, the generalized expression of the limiting length of fixed windows at which half width of the window function in time domain equals to the NBW in frequency domain can be obtained for above reported window functions from (6), (11), (15), (19) respectively at $\alpha=\pi / 2$ as-

$$
\begin{aligned}
& \frac{N+1}{2(M+N)}=\frac{p}{N} \quad \text { if } N \text { is odd } \\
& \frac{N}{2(M+N)}=\frac{p}{N} \quad \text { if } N \text { is even }
\end{aligned}
$$


where, $M$ is number of padded samples in either sides of the window function to increase resolution and $p=1$ for RW, 2 for GHW \& TW and 3 for BW.

The mathematical relation given in (22) is a quadratic equation and its positive root, which defines the limiting length of window functions, is given as-

$$
N=p+\sqrt{p+\frac{2 M}{p}}
$$

The value of $N$ given by (23) is the limiting length of the fixed window functions above which the NBW expands by decreasing the value of FRFT order $a$ from 1 to zero. The obtained limiting length for $\mathrm{RW}(p=1)$ with padded zeroes $M=512$ is $N=32$ at which NBW and half width of RW becomes equal as shown in Figure 8. It can be also observed from Figures. 9-10 that the NBW of RW reduces by decreasing the value of $a$ from 1 to zero if the number of samples in the window are less than 32 and expands if the samples are more than 32 Similar behavior has been also observed for other fixed windows as shown in Figures.11-16. Figures 9-16 have been shown from 0 to 8 bins only in place of actual range 0 to $N / 2$ bins for the sake of more clarity.

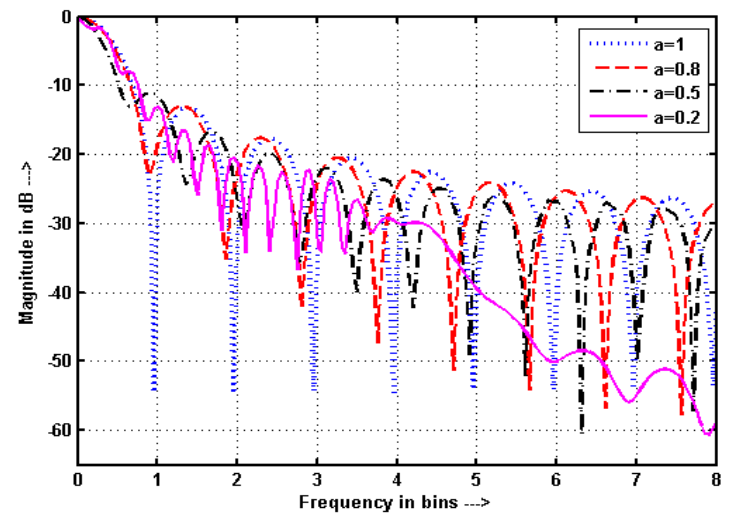

Figure 9. Frequency response of RW with a for $\mathrm{N}=27$ and $\mathrm{M}$ $=512$.

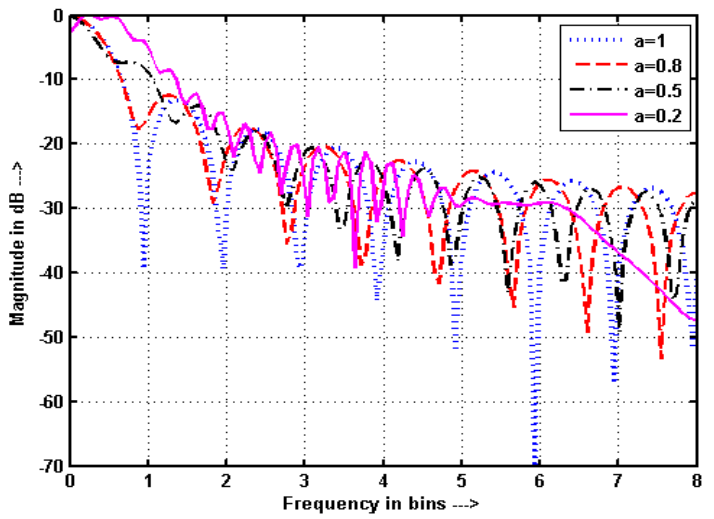

Figure 10. Frequency response of RW with a for $\mathrm{N}=37$ and $\mathrm{M}$ $=512$

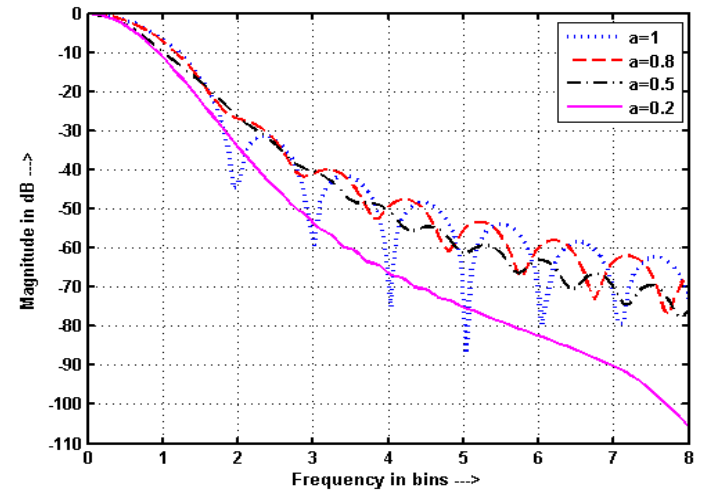

Figure 11. Frequency response of $\mathrm{HW}$ with a for $\mathrm{N}=43$ and $\mathrm{M}=512$

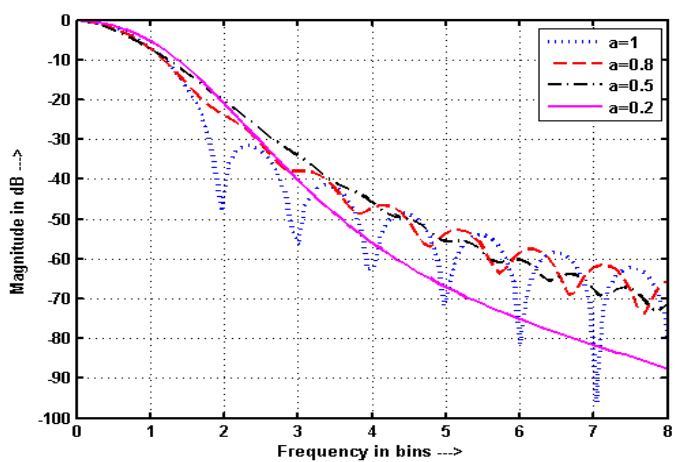

Figure 12. Frequency response of $\mathrm{HW}$ with a for $\mathrm{N}=53$ and $\mathrm{M}=512$

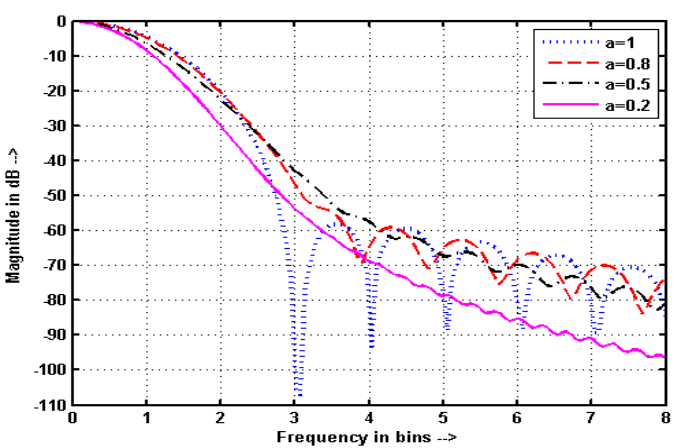

Figure 13. Frequency response of $\mathrm{BW}$ with a for $\mathrm{N}=70$ and $\mathrm{M}$ $=1024$

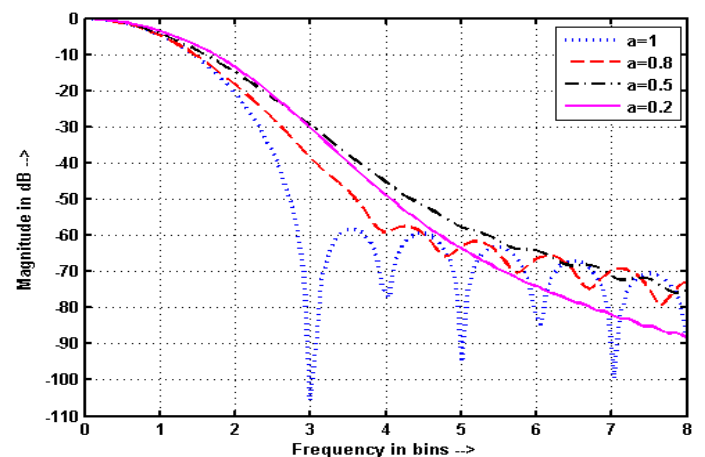

Figure 14. Frequency response of $\mathrm{BW}$ with a for $\mathrm{N}=90$ and $\mathrm{M}$ $=1024$

I.J. Image, Graphics and Signal Processing, 2014, 2, 1-13 


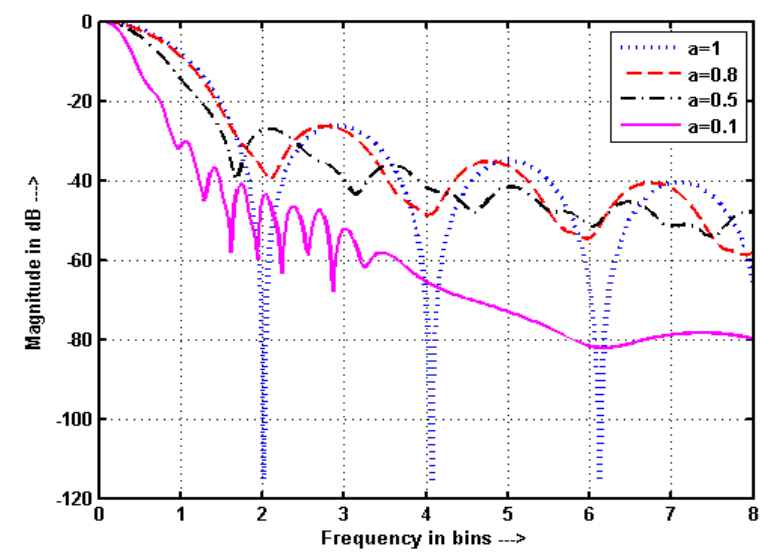

Figure 15. Frequency response of BW with a for $\mathrm{N}=39$ and $\mathrm{M}=512$

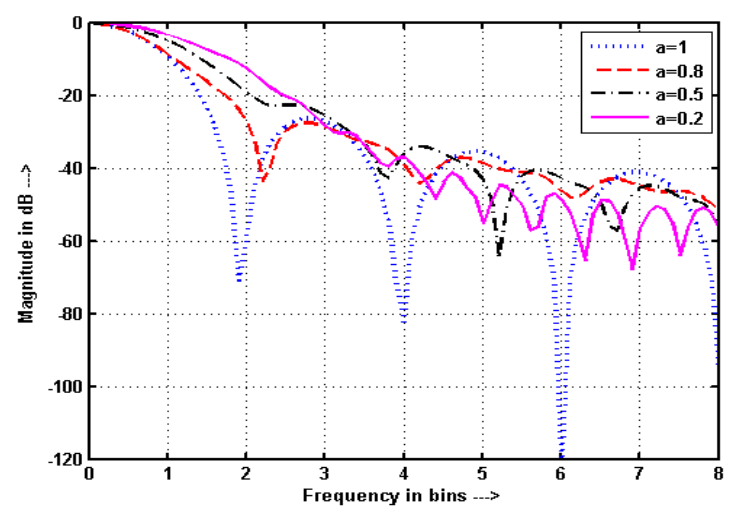

Figure 16. Frequency response of $\mathrm{BW}$ with a for $\mathrm{N}=57$ and $\mathrm{M}$ $=512$

\section{IV.TUNING OF FIR FILTERS}

The TBW of window based FIR filters is proportional to the filter order. Thus, it can be varied by the variation of filter order which needs the re-computation of the impulse response coefficients of the filter. An alternative FRFT based method to tune the TBW of these filters using ideal impulse response has been developed by Sharma et al. [7] which eliminates the recomputation problem. In this work, in place of ideal impulse response of the filter, realistic impulse response $h(n)$ has been used and characteristics of FIR filter i. e. TBW, Stopband Attenuation (SBA) and Passband Ripple (PBR) are being varied by varying the frequency response of window function $w(n)$ with the variation of FRFT order $a$. To obtain the filtered output in frequency domain the circularly convolved output of FFT of impulse response $h(n)$ i.e. $H(k)$ and FRFT of $w(n)$ is multiplied by FFT of input signal $x(n)$ i.e. $X(k)$ as shown in Figure 17.

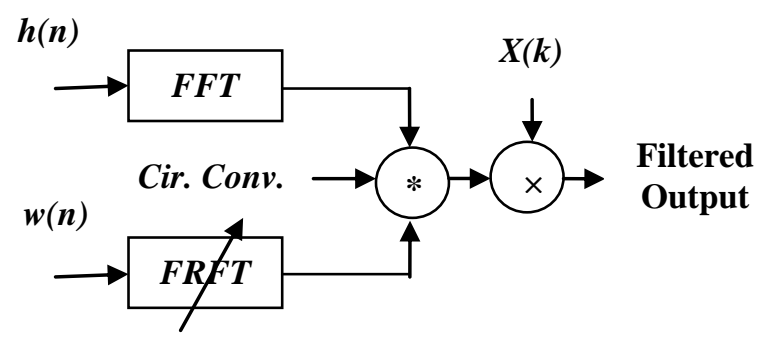

Figure 17. Tuning scheme of FIR filters using FRFT

The tuning of frequency responses of FIR filters using Hanning and Blackman windows are shown in Figures. 18-21. It has been observed from these responses that, by varying FRFT order from 1 to 0 , the TBW of FIR filters decreases according to $f_{c}+u_{F T} \sin (\alpha)$ approximately, where $f_{c}$ is the cutoff frequency of the filter, and SBA and PBR increases if the filter order is less than (23) while behavior of these parameters becomes opposite if the order of filter is greater than (23). Theoretically, this happens because if the length of window is equal to (23) then the window spans in time domain and frequency domain are equal at FRFT order 1 while time span becomes less than frequency span and tends toward impulse function if the length of window is less than (23) when FRFT order decreases from 1 to 0 and it becomes vice versa for the window length greater than (23). This behavior of window functions is directly affecting parameters of FIR filters designed using these fixed window functions. Thus it can be said that parameters of windowed FIR filters can be tuned using FRFT up to the filter designed without windowing i. e. impulse response method without re-computing the filter coefficients.
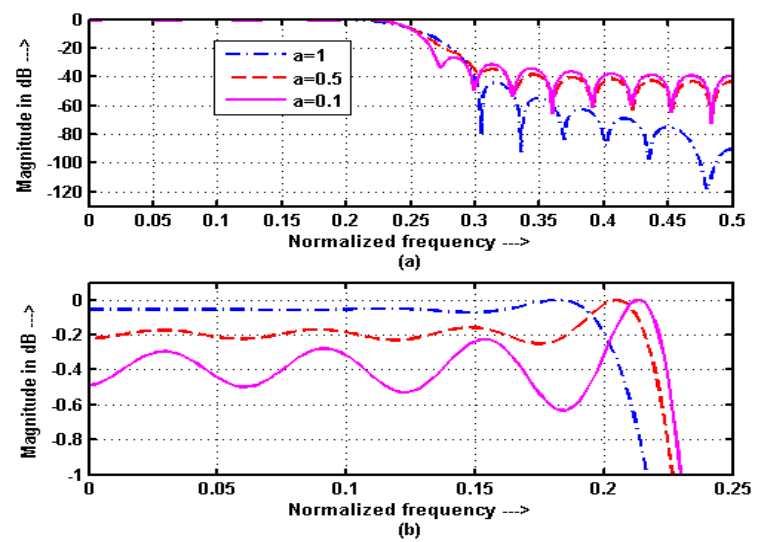

Figure 18. Frequency responses of LPF using HW with $\mathrm{fp}=$ 0.2 , fs $=0.3, \mathrm{~N}=31$ (a) Comparative frequency responses (b) expanded view of passband 

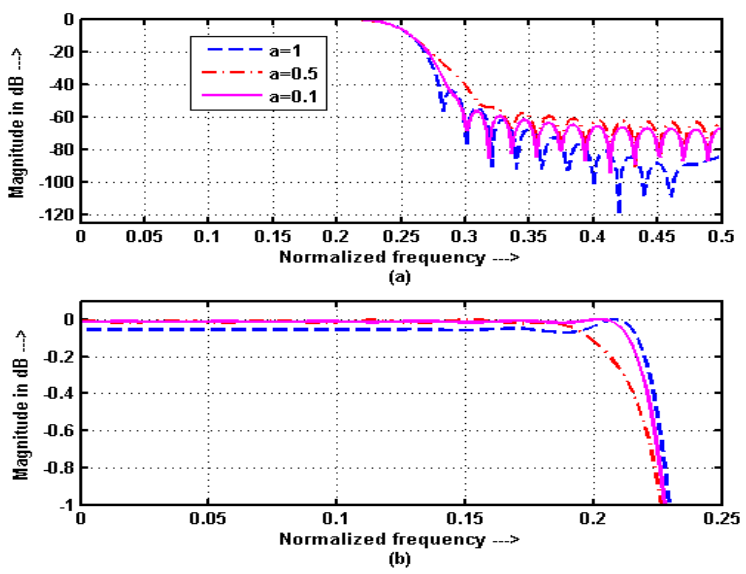

Figure 19. Frequency responses of LPF using HW with $\mathrm{fp}=$ $0.2, \mathrm{fs}=0.3, \mathrm{~N}=51$ (a) Comparative frequency responses (b) expanded view of passband
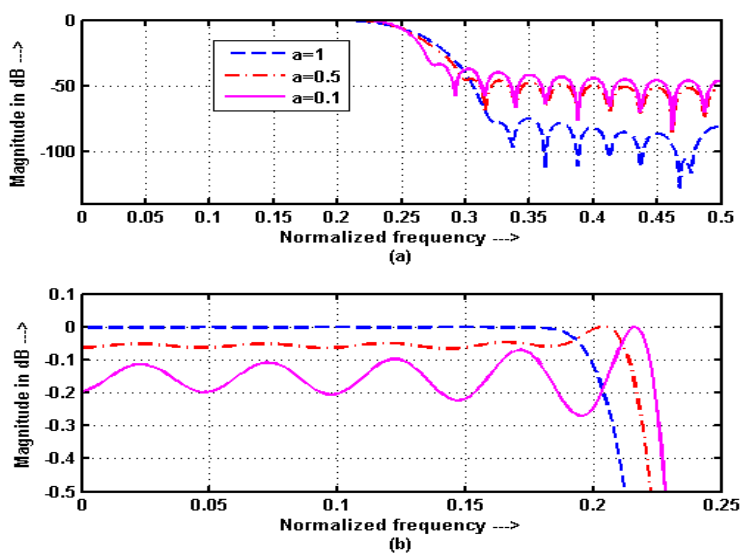

Figure 20. Frequency responses of LPF using BW with $\mathrm{fp}=$ $0.2, \mathrm{fs}=0.3, \mathrm{~N}=41$ (a) Comparative frequency responses (b) expanded view of passband
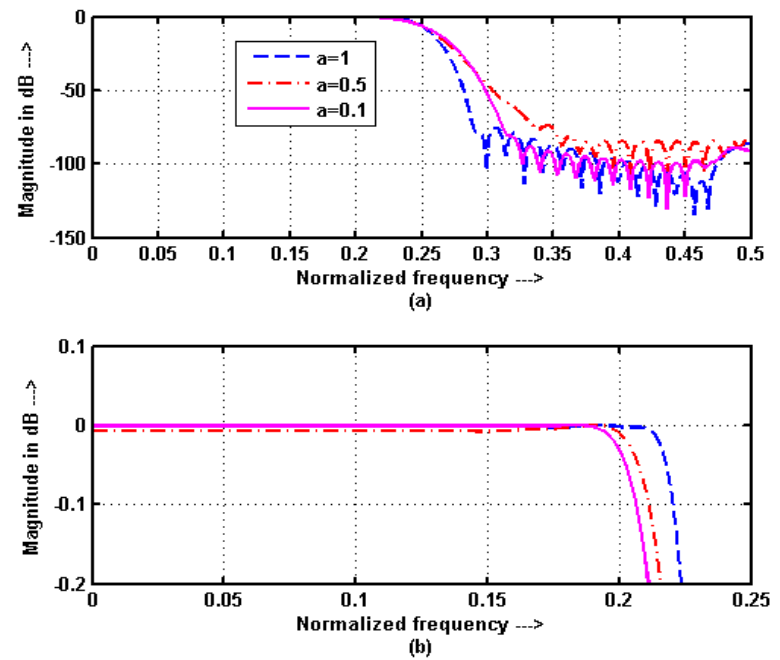

Figure 21. Frequency responses of LPF using BW with $\mathrm{fp}=$ $0.2, \mathrm{fs}=0.3, \mathrm{~N}=71$ (a) Comparative frequency responses (b) expanded view of passband

\section{CONCLUSION}

The analytical expressions have been derived for four popular fixed window functions. It has been observed that the window parameters like NBW, HMLW and MSLL may have one more controlling variable (FRFT order $a$ ). The derived expressions have been verified with simulation studies in all situations. Thus, it can be said that these windows can be considered as variable windows in time-frequency plane, keeping their time domain nature unchanged, which are inherently fixed in nature. The derived expressions can be considered very useful in more efficient design of window based digital FIR filters and spectral analysis. The obtained adaptability in the fixed windows has been exploited in the tuning of the parameters of FIR filters.

\section{REFERENCES}

[1] L.R. Rabiner, B. Gold. Theory and Applications of Digital Signal Processing, Englewood Cliffs, NJ: Prentice-Hall, 1975.

[2] F. J. Harris. On the use windows for harmonic analysis with discrete Fourier transform. Proc. IEEE, 1978, 66(1):51-83.

[3] L. B. Almeida. The fractional Fourier transform and time-frequency representation. IEEE Trans., Signal Processing, 1994, 42(11): 3084-3093.

[4] V. Namias. The fractional order Fourier transform and its application to quantum mechanics. J. Inst. Math., 1980, Appl. 25:241-265.

[5] H. M. Ozaktas, Z. Zalevsky, M. A. Kutey. The Fractional Fourier transform with Applications in Optics and Signal processing. 1st ed., Wiley, Newyork, 2001.

[6] S. Kumar, K. Singh, R. Saxena. Analysis of Dirichlet and Generalized Hamming window functions in fractional Fourier transform domain. Signal Processing, 2011, 91:600-606.

[7] S. N. Sharma, R. Saxena, S. C. Saxena. Tuning of FIR filter transition bandwidth using fractional Fourier transform. Signal Processing, 2007, 87: 3147-3154.

[8] R. Saxena. Synthesis and characterization of new window families with their applications. Ph. D. Dissertation, Department of Electronics and Computer Engineering, University of Roorkee (Presently IIT Roorkee), India, 1996.

[9] D. L. Donoho, P.B. Stark. Uncertainty principles and signal recovery. SIAM J. Apll. Maths. 1989, 49(3): 906-931.

[10] J. G. Proakis, D. G. Manolakis. Digital signal processing, principles, algorithms, and applications. Prentice-Hall, 2007.

[11] J. K. Gautam, A. Kumar, R. Saxena. On the modified Bartlett-Hanning window (family). IEEE Trans., Signal Processing, 1996, 44 (8): 2098-2102.

[12] N. C. Geckinli, D. Yavuz. Some novel windows and a concise tutorial comparison of window families. IEEE Trans., Acoustics, speech, and 
Signal Processing, 1978, 26 (6): 501-507.

[13] A. Antoniou. Digital signal processing: signals, systems, and filters. McGraw-Hill, 2005.

\section{AUTHOR'S PROFILE}

Rahul Pachauri is an Assistant Professor at Jaypee University of Engineering \& Technology, Guna (M.P.) India. He is currently working toward the Ph.D. degree in the Electronics \& Communication Engineering. His research interests include digital signal processing and wireless communication.

Rajiv Saxena is currently working as Professor and Head in the Department of Electronics \& Communication Engineering, Jaypee University of
Engineering \& Technology, Guna (M.P.) India. His teaching and research interest includes digital signal processing, communication systems, integral transform, digital image processing and mobile communication. He has 29 years teaching experience. He has guided $13 \mathrm{Ph}$. D. scholars and published more than 45 research papers in the various international and national referred journals.

S. N. Sharma is currently working as Associate Professor and Head in the Department of Electronics \& Communication Engineering, Samrat Ashok Technological Institute, Vidisha (M.P.), India. His teaching and research interests include digital signal processing, genomic signal processing, fractional Fourier transform, bio signal processing. He has 18 years teaching experience. He has published 12 research papers in various reputed journals.

\section{APPENDIX-1}

The RW can be expressed in FRFT domain by using (1) as-

$$
W_{R}(u)=\sqrt{\frac{1-i \cot (\alpha)}{2 \pi}} \exp \left[i \frac{u^{2}}{2} \cot (\alpha)\right] \int_{-\tau / 2}^{\tau / 2} 1 \times \exp \left[i \frac{t^{2}}{2} \cot (\alpha)-i u t \operatorname{cosec}(\alpha)\right] d t
$$

BY REWRITING THE EXPRESSION FOR $W_{R}(U)-$

$$
W_{R}(u)=\sqrt{\frac{1-i \cot (\alpha)}{2 \pi}} \exp \left[i \frac{u^{2}}{2} \cot (\alpha)\right] \times \int_{-\tau / 2}^{\tau / 2} \exp \left\{\frac{i}{2} \cot (\alpha)\left[\{t-u \sec (\alpha)\}^{2}-\{u \sec (\alpha)\}^{2}\right] d t\right.
$$

Substituting, $\{t-u \sec (\alpha)\}=R$ and changing the limits of the integration of (A1.2),

$W_{R}(u)=\sqrt{\frac{1-i \cot (\alpha)}{2 \pi}} \exp \left[i \frac{u^{2}}{2} \cot (\alpha)\right] \times \int_{-0.5 \tau-u \sec (\alpha)}^{0.5 \tau-u \sec (\alpha)} \exp \left[\frac{i}{2} \cot (\alpha) R^{2}\right] d R$

Now, solving the integration, following expression results for RW in FRFT domain -

$$
\begin{aligned}
W_{R}(u) & =-\sqrt{\frac{1-i \cot (\alpha)}{8}} \exp \left[-\frac{i}{2} u^{2} \tan (\alpha)\right] \times \\
& \left\{\operatorname{erfi}\left[\frac{1+i}{2} \sqrt{\cot (\alpha)}\left\{\frac{\tau}{2}-u \sec (\alpha)\right\}\right]-\operatorname{erfi}\left[\frac{1+i}{2} \sqrt{\cot (\alpha)}\left\{-\frac{\tau}{2}-u \sec (\alpha)\right\}\right]\right\}
\end{aligned}
$$

where, erfi $(z)$ is an analytical function of $z$ which is defined in the whole complex $z$-plane. 


\section{APPENDIX-2}

The differentiation of $W_{R}(u)$ with respect to $u$ is given by-

$$
\begin{aligned}
& \frac{d}{d u}\left|W_{R}(u)\right|=\frac{2}{\sqrt{\pi}}\left|\sqrt{\frac{1-i \cot (\alpha)}{8}}\right| \times \\
&\left.\mid \exp \left[\frac{1+i}{2} \sqrt{\cot (\alpha)}\left\{\frac{\tau}{2}-u \sec (\alpha)\right\}\right]^{2}-\exp \left[\frac{1+i}{2} \sqrt{\cot (\alpha)}\left\{-\frac{\tau}{2}-u \sec (\alpha)\right\}\right]\right]^{2} \mid
\end{aligned}
$$

The position of nulls can be obtained by equating the differentiation of $W_{R}(u)$ to zero as-

$$
\left|\exp \left[\frac{1+i}{2} \sqrt{\cot (\alpha)}\left\{\frac{\tau}{2}-u \sec (\alpha)\right\}\right]^{2}-\exp \left[\frac{1+i}{2} \sqrt{\cot (\alpha)}\left\{-\frac{\tau}{2}-u \sec (\alpha)\right\}\right]^{2}\right|=0
$$

The expression (A2.2) after further simplification can be written as-

$$
\left|\exp \left[0.5 i \cot (\alpha)\left\{0.25 \tau^{2}+u^{2} \sec ^{2}(\alpha)\right\}\right] \times\right| \exp [-0.5 i u \tau \operatorname{cosec}(\alpha)]-\exp [0.5 i u \tau \operatorname{cosec}(\alpha)] \mid=0
$$

Equation (A2.3) when solved for $u$ involves following steps-

$$
\begin{gathered}
\left|2 \exp \left[0.5 i \cot (\alpha)\left\{0.25 \tau^{2}+u^{2} \sec ^{2}(\alpha)\right\}\right]\right| \times|\sin \{0.5 u \tau \operatorname{cosec}(\alpha)\}|=0 \\
|0.5 u \tau \operatorname{cosec}(\alpha)|=n \pi \quad \text { for } \quad n=0,1,2 \ldots \\
u=\left|\frac{2 n \pi \sin (\alpha)}{\tau}\right| \quad \text { for } \quad n=0,1,2 \ldots
\end{gathered}
$$

\section{APPENDIX-3}

The GHW for $b=0.5$ can be expressed in FRFT domain as-

$$
\begin{aligned}
W_{H}(u) & =c_{1}\left[c_{2}\left\{\operatorname{erfi}\left[c_{3}\{u-0.5 \cos (\alpha)\}\right]-\operatorname{erfi}\left[c_{3}\{u+0.5 \cos (\alpha)\}\right]\right\}\right. \\
& +c_{4}\left\{\operatorname{erfi}\left[c_{3}\{u+0.5 \cos (\alpha)+2 \pi \sin (\alpha)\}\right]-\operatorname{erfi}\left[c_{5}\{u-0.5 \cos (\alpha)+2 \pi \sin (\alpha)\}\right]\right\} \\
& \left.+c_{6}\left\{\operatorname{erfi}\left[c_{3}\{-u+0.5 \cos (\alpha)+2 \pi \sin (\alpha)\}\right]-\operatorname{erfi}\left[c_{7}\{-2 \pi \sec (\alpha)+\operatorname{cosec}(\alpha)[0.5+u \sec (\alpha)]\}\right]\right\}\right]
\end{aligned}
$$

where,

$$
\begin{array}{llrl}
c_{1}=0.3536(-1)^{3 / 4} e^{-\frac{i}{2}\left(u^{2}+8 \pi^{2}\right) \tan (\alpha)} \sqrt{\tan (\alpha)-i} ; & c_{2}=2 b e^{4 i \pi^{2} \tan (\alpha)} \\
c_{3}=\frac{(-1)^{1 / 4}}{\sqrt{\sin (2 \alpha)}} ; & c_{4}=(-1+b) \mathrm{e}^{2 \mathrm{i} \pi \sec (\alpha)[-u+\pi \sin (\alpha)]} ; & c_{5}=(0.5+0.5 \mathrm{i}) / \sqrt{0.5 \sin (2 \alpha)}
\end{array}
$$




$$
\begin{gathered}
c_{6}=(-1+b) \mathrm{e}^{2 \mathrm{i} \pi \sec (\alpha)[u+\pi \sin (\alpha)]} \quad \text { and } \quad c_{7}=-0.5(-1)^{1 / 4} \sqrt{\sin (2 \alpha)} \\
c_{1}\left[\begin{array}{l}
c_{2}\left\{\begin{array}{l}
\operatorname{erfi}\left[c_{3}\left\{1.175 u_{F T} \sin (\alpha)-0.5 \cos (\alpha)\right\}\right] \\
\left.-\operatorname{erfi}\left[c_{3}\left\{1.175 u_{F T} \sin (\alpha)+0.5 \cos (\alpha)\right\}\right]\right\}
\end{array}\right\} \\
+c_{4}\left\{\begin{array}{l}
\operatorname{erfi}\left[c_{3} \sin (\alpha)\left\{1.175 u_{F T}+0.5 \cot (\alpha)+2 \pi\right\}\right] \\
-\operatorname{erfi}\left[c_{5} \sin (\alpha)\left\{1.175 u_{F T}-0.5 \cot (\alpha)+2 \pi\right\}\right]
\end{array}\right\} \\
+c_{6}\left\{\begin{array}{l}
\operatorname{erfi}\left[c_{3} \sin (\alpha)\left\{-1.175 u_{F T}+0.5 \cot (\alpha)+2 \pi\right\}\right] \\
-\operatorname{erfi}\left[c_{7} \sec (\alpha)\left\{-2 \pi+0.5 \cot (\alpha)+1.175 u_{F T}\right\}\right]
\end{array}\right]
\end{array}\right]
\end{gathered}
$$

\section{APPENDIX-4}

The FRFT domain expression for BW can be written as-

$$
W_{B}(u)=c_{1}\left[\begin{array}{l}
c_{2}\left(\operatorname{erfi}\left[-c_{7}(-2 u+\cos [\alpha])\right]-\operatorname{erfi}\left[c_{8}(2 u+\cos [\alpha])\right]\right) \\
+c_{3}\left(\operatorname{erfi}\left[c_{8}\left(2 u-\cos [\alpha]+c_{9}\right)\right]-\operatorname{erfi}\left[c_{8}\left(2 u+\cos [\alpha]+c_{9}\right)\right]\right) \\
+c_{4}\left(\operatorname{erfi}\left[c_{10}(\operatorname{cosec}[\alpha]-4 \pi \operatorname{Sec}[\alpha]+4 u \operatorname{cosec}[2 \alpha])\right]-\operatorname{erfi}\left[c_{8}\left(-2 u+\cos [\alpha]+c_{9}\right)\right]\right) \\
+c_{5}\left(\operatorname{erfi}\left[c_{10}(\operatorname{cosec}[\alpha]-8 \pi \sec [\alpha]+4 u \operatorname{cosec}[2 \alpha])\right]-\operatorname{erfi}\left[c_{8}\left(-2 u+\cos [\alpha]+2 c_{9}\right)\right]\right) \\
+c_{6}\left(\operatorname{erfi}\left[c_{8}\left(2 u-\cos [\alpha]+2 c_{9}\right)\right]-\operatorname{erfi}\left[c_{8}\left(2 u+\cos [\alpha]+2 c_{9}\right)\right]\right)
\end{array}\right]
$$

where,

$$
\begin{aligned}
& c_{1}=0.25(-1)^{3 / 4} \mathrm{e}^{-\mathrm{i}\left(-0.5 u^{2} \cot [\alpha]\left(1-\sec ^{2}[\alpha]\right)+4 \pi \sec [\alpha]\left(u+\pi \sin [\alpha]\left(1+\operatorname{cosec}^{2}[\alpha]\right)\right)\right)} \sqrt{\tan [\alpha]-\mathrm{i}} \\
& c_{2}=0.84 \mathrm{e}^{-4 \mathrm{i} \pi \operatorname{cosec}[2 \alpha](-3 \pi+\pi \cos [2 \alpha]-2 u \sin [\alpha])} ; \quad c_{3}=0.5 \mathrm{e}^{-2 \mathrm{i} \pi \operatorname{cosec}[2 \alpha](-5 \pi+\pi \cos [2 \alpha]-2 u \sin [\alpha])} ; \\
& c_{4}=0.5 \mathrm{e}^{-2 \mathrm{i} \pi \operatorname{cosec}[2 \alpha](-5 \pi+\pi \cos [2 \alpha]-6 u \sin [\alpha])} ; \quad \quad c_{5}=0.08 \mathrm{e}^{4 \pi \mathrm{i}(\pi \cot [\alpha]+2 u \sec [\alpha])} ; \\
& c_{6}=0.08 \mathrm{e}^{4 \mathrm{i} \pi^{2} \cot [\alpha]} ; \quad c_{7}=(-1)^{1 / 4} /(2 \sqrt{\sin [2 \alpha]}) ; \quad c_{8}=(0.25+0.25 \mathrm{i}) / \sqrt{\cos [\alpha] \sin [\alpha]} ; \\
& c_{9}=4 \pi \sin [\alpha] ; \quad \text { and } \quad c_{10}=-0.25(-1)^{1 / 4} \sqrt{\operatorname{Sin}[2 \alpha]}
\end{aligned}
$$

Therefore, the MSLL of BW in FRFT domain can be written as-

$$
\begin{aligned}
|M S L L|_{B}= & \mid c_{1}\left[c_{2}\left(\operatorname{erfi}\left[-c_{7}\left(-2.36 u_{F T} \sin [\alpha]+\cos [\alpha]\right)\right]-\operatorname{erfi}\left[-c_{8}\left(2.36 u_{F T} \sin [\alpha]+\cos [\alpha]\right)\right]\right)\right. \\
& c_{3}\left(\operatorname{erfi}\left[c_{8}\left(2.36 u_{F T} \sin [\alpha]-\cos [\alpha]+c_{9}\right)\right]-\operatorname{erfi}\left[c_{8}\left(2.36 u_{F T} \sin [\alpha]+\cos [\alpha]+c_{9}\right)\right]\right)+ \\
& c_{4}\left(\operatorname{erfi}\left[c_{10}\left(\operatorname{cosec}[\alpha]-4 \pi \sec [\alpha]+2.36 u_{F T} \sec [\alpha]\right)\right]-\operatorname{erfi}\left[c_{8}\left(-2.36 u_{F T} \sin [\alpha]+\cos [\alpha]+c_{9}\right)\right]\right)+ \\
& c_{5}\left(\operatorname{erfi}\left[c_{10}\left(\operatorname{cosec}[\alpha]-8 \pi \sec [\alpha]+2.36 u_{F T} \sec [\alpha]\right)\right]-\operatorname{erfi}\left[c_{8}\left(-2.36 u_{F T} \sin [\alpha]+\cos [\alpha]+2 c_{9}\right)\right]\right)+ \\
& \left.c_{6}\left(\operatorname{erfi}\left[c_{8}\left(2.36 u_{F T} \sin [\alpha]-\cos [\alpha]+2 c_{9}\right)\right]-\operatorname{erfi}\left[c_{8}\left(2.36 u_{F T} \sin [\alpha]+\cos [\alpha]+2 c_{9}\right)\right]\right)\right] \mid
\end{aligned}
$$




\section{APPENDIX-5}

The TW can be expressed in fractional domain as-

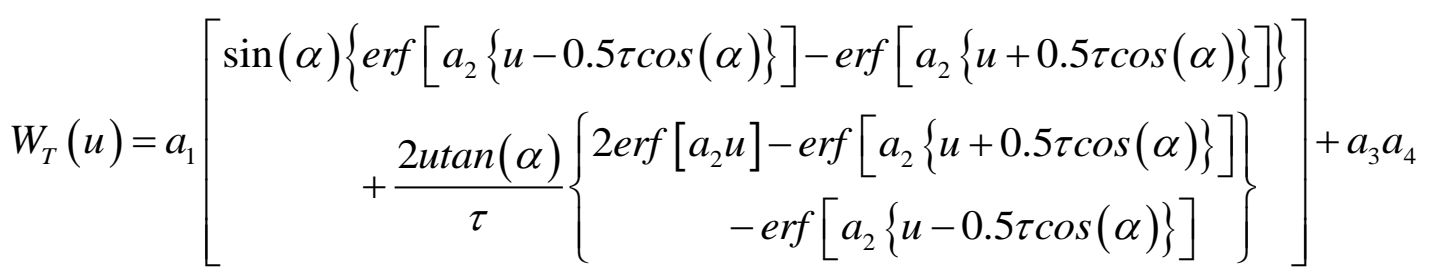

where,

$$
\begin{aligned}
& a_{1}=\sqrt{\frac{i+\cot (\alpha)}{2 \sin (2 \alpha)} \exp \left[i \frac{u^{2}}{2} \cot (\alpha)\right] ;} \quad a_{2}=-\sqrt{-i \operatorname{cosec}(2 \alpha)} \\
& a_{3}=\frac{2 \tan (\alpha)}{\tau} \sqrt{\frac{1-i \cot (\alpha)}{2 \pi}} \exp \left[i \frac{u^{2}}{2} \cot (\alpha)\right] ; \quad \text { and } \\
& a_{4}=-2 i \exp \left\{i u^{2} \operatorname{cosec}(\alpha)\right\}+i\left\{\exp \left[i \frac{u^{2}}{2}\{0.5 \tau-u \sec (\alpha)\} \cot (\alpha)\right]+\exp \left[i \frac{u^{2}}{2}\{0.5 \tau+u \sec (\alpha)\} \cot (\alpha)\right]\right\}
\end{aligned}
$$

The expression for MSLL of TW in FRFT domain can be written as-

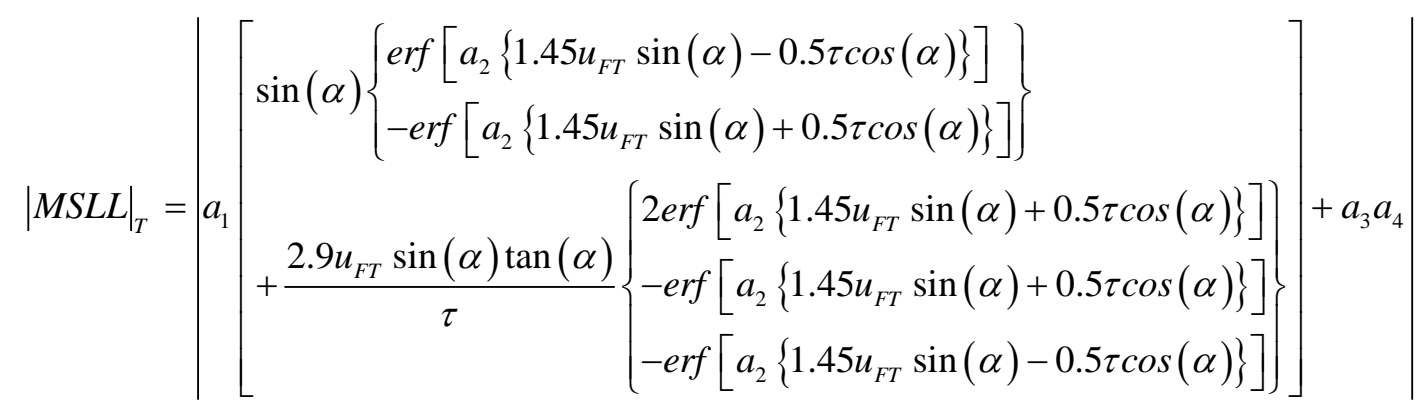

